\title{
Repeat polymorphism analysis for examining genetic variability and the implications for specific traits in dog breeds
}

\author{
Jungwoo Eo and Heui-Soo Kim* \\ Department of Biological Sciences, College of Natural Sciences, Pusan National University, \\ Busan 609-735, Republic of Korea
}

(Received 23 October 2013, accepted 6 January 2014)

\begin{abstract}
Genetic variations of functional genes in various animal species have been previously examined to understand the relationship between genotypes and phenotypes. Repeat polymorphism can be found in not only coding sequences but also untranslated regions of the protein-coding genes, affecting gene regulation via a variety of mechanisms. In this respect, repeat polymorphisms including microsatellites, variable number of tandem repeats (VNTRs), and short interspersed nuclear elements (SINEs) in relation to functional genes contribute to recognition of genetic or phenotypic variation and individual identification. These elements are biologically valuable markers for studies on association between genetic makeup and behavioral patterns in dog breeds. Hence, to examine the effect of genotype on behavior, studies on this combination are certainly important. Hence, this review could cast light on the functional roles of repeat polymorphisms in dog behavior and breed variation.
\end{abstract}

Key words: dogs, functional genes, microsatellites, short interspersed elements (SINEs), variable number of tandem repeats (VNTRs)

\section{INTRODUCTION}

Genetic variation in functional genes has been a focal point for understanding the relationship between genotypes and behavioral phenotypes in various animal species. Genetic variation plays a significant role in the development of inherited features and the evolution of domestic breeds. With respect to phenotypic diversity, the dog is ranked as the most unique domesticated animal (Parker et al., 2004). Single-locus SNP analysis of the genetic relationships among dog breeds indicated that the consensus tree clearly supported the basic division between wolves and domestic dogs (vonHoldt et al., 2010). However, the classification of breeds into functional and phenotypic groups is not true of all breed groupings. In order to solve this problem, an approach for genetic identification of multifactorial traits was employed, involving an association analysis of candidate genes in dog populations.

Repeat polymorphism in genes is associated with diverse phenotypes by influencing the gene function. Analysis on the repeat polymorphism leads us to identify differences between various dog breeds based on genetic

\footnotetext{
Edited by Yoko Satta

* Corresponding author. E-mail: khs307@pusan.ac.kr
}

diversity. It can also help us to understand the relationship between genetic features and behavioral phenotype in dogs. Dynamic potential of repeat polymorphism in dog as well as human have been well studied. Among repeat elements that are classified based on their structure and size of repeat unit, microsatellites in mammalian genomes consist of the simplest tandem repeats of short units, which could be effective genetic markers for dog breed phylogeny, genetic variation, and quality control (Irion et al., 2003; Ellegren, 2004). An investigation of the trinucleotide (CAG) repeat encoding glutamine in exon 1 of the androgen receptor $(A R)$ gene indicated that polymorphisms of the CAG microsatellite are associated with aggression in Japanese Akita Inu (Konno et al., 2011). A relatively short CAG repeat sequence enhances the transcriptional activity of $A R$ by promoting the interaction between the receptor and co-activator (Chamberlain et al., 1994).

In addition, an association study between behavior and a repeat polymorphism in intron 2 of the canine dopamine receptor D4 (DRD4) gene demonstrated a significant association between social impulsivity and intronic VNTRs (variable number of tandem repeats) of the $D R D 4$ gene in German Shepherds (Hejjas et al., 2009). It was also reported that a repeat polymorphism in the tyrosine hydroxylase $(T H)$ gene is linked to activity-impulsivity in 
German Shepherds (Kubinyi et al., 2012). The VNTR polymorphism in the $D R D 4, T H$, dopamine $\beta$-hydroxylase $(D B H)$, and dopamine transporter $(D A T)$ genes has been investigated among four dog breeds and European Grey Wolves in order to understand its relationship with the activity-impulsivity phenotype (Hejjas et al., 2007).

How could the repeat polymorphism in functional genes be associated with phenotype? The polymorphic repeat elements could be found in coding sequence (CDS) or untranslated regions (UTRs) of functional genes, and have dynamic impacts on gene regulation (Fig. 1). The repeat polymorphism can influence gene expression by acting as a cis-element, and in mRNA processing such as splicing and adenylation. However, the mechanisms by which the repeat elements are involved in gene regulation at diverse steps remain unexplored. The $5^{\prime}$ UTR is especially thought to be a crucial region to regulate gene expression through various mechanisms (Chatterjee and Pal, 2009).

Many studies in human suggest that repeat polymorphism in the $5^{\prime}$ UTR could have an effect on gene expression. For example, a VNTR in promoter region monoamine oxidase A (MAOA) gene affects its expression by altering transcriptional activities of the $M A O A$ promoter depending on the number of repeats in human (Deckert et al., 1999). In case of human sirtuin 3 (SIR3) gene, a VNTR polymorphism in intron 5 has been investigated, demonstrating an allele-specific enhancer activity in vitro (Bellizzi et al., 2005). As for studies on relationship with splicing events, a polymorphic GT simple repeat in intron 2 of human cardiac $\mathrm{Na}^{+} \mathrm{Ca}^{2+}$ exchanger 1 (NCX1) gene exhibits variable length, and acts as a strong intronic splicing enhancer that could reg- ulate the NCX1 expression, possibly by controlling tissuespecific alternative splicing (Gabellini, 2001).

Here, we reviewed repeat polymorphisms including microsatellites, VNTRs, and short interspersed nuclear elements (SINEs) associated with functional genes, focusing on relationship with behavioral variation and individual identification. We further suggest application of these tools as practically useful markers for association genetic studies of dog breeds.

\section{MICROSATELLITE POLYMORPHISM AND SNP IN DOGS}

Dogs play a number of important roles in modern society and are an ideal animal for elucidating the genomic features of behavioral traits (Spady and Ostrander, 2008). Microsatellites, tandem repeats of short units (1-6 bp in length), tend to expand with higher copy numbers because the length is likely to be altered during DNA replication at a higher rate than the average rate of point mutations (Ellegren, 2004; Payseur et al., 2011; Sun et al., 2012). Microsatellite loci could serve as suitable markers for parentage tests and individual identification to achieve reliable pedigree verification of dog breeds (Kang et al., 2009). The technique using various microsatellite markers simultaneously could be a more costeffective, more accurate, and faster way to infer genetic variation and pedigree information. As the multiplex PCR technique is useful for analyzing multiple alleles in dog breeds, experimental studies based on multiplex PCR with microsatellite markers have been reported (Kim et al., 2001; Takeuchi et al., 2005, 2009b). Genetic variability and structure in East Asian dog populations were ana-

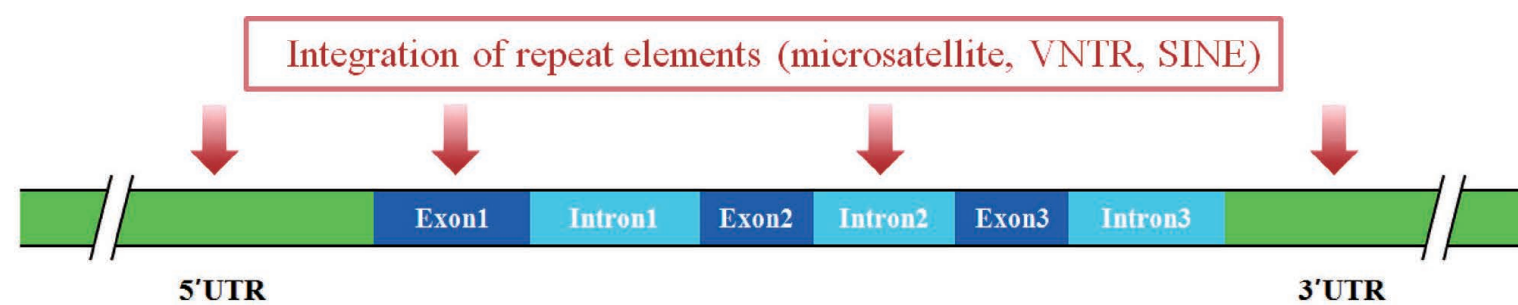

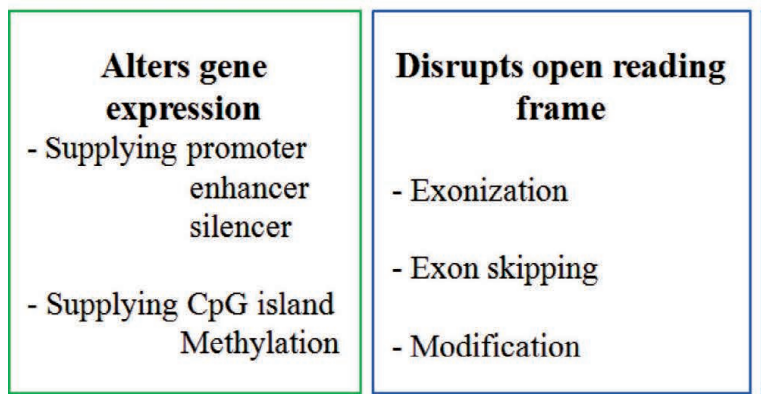
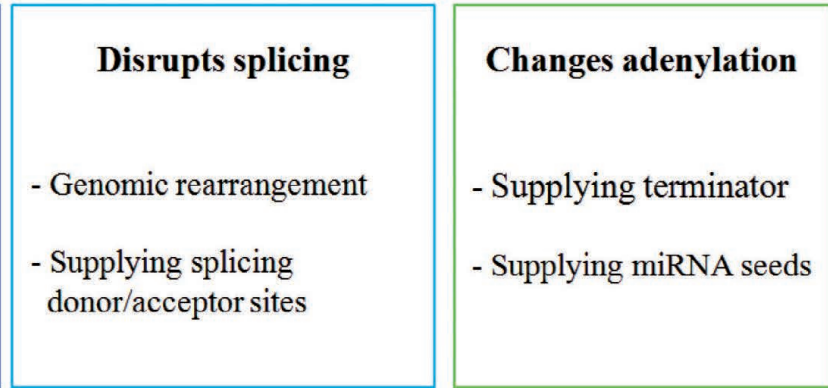

Fig. 1. Schematic representation of gene dynamics by repeat elements (microsatellite, VNTR, and SINE) and their plausible biological roles in functional genes. The repeat elements located in various genetic regions (exons, introns, $5^{\prime}$ UTR, and $3^{\prime}$ UTR) can influence gene regulation through diverse mechanisms. 
lyzed by eight microsatellites, demonstrating that Korean native dogs formed the closest groups and showed a close relationship to the Eskimo dogs. These results indicated that allele frequency and heterozygosity were suitable for individual identification and paternity diagnosis (Kim et al., 2001). Gene-associated microsatellites have been identified. Two polymorphic microsatellites in canine Monoamine Oxidase A (MAOA) gene, encoding a mitochondrial enzyme that degrades the neurotransmitters, have been reported (Klukowska et al., 2004). As a point mutation of human MAOA is associated with abnormal behavior (Brunner et al., 1993), these polymorphisms could be involved in behavioral changes in dogs. Additionally, a microsatellite polymorphism between canine behavioral traits and genetic variability is expected to be associated with some types of aggressive behavior in the Shiba Inu (Takeuchi et al., 2009b). In the case of the solute carrier family 1 member 2 (SLC1A2) gene, the TT genotype located in coding region was more active than the TC or CC genotypes in dog behavior (Takeuchi et al., 2009a). As a study for the genetic background of canine behavioral traits, polymorphism in the $T H$ and $D B H$ gene, which encode biosynthetic enzymes producing catecholamine neurotransmitters biosynthetic enzymes, were reported (Takeuchi et al., 2005). The C97T polymorphism in $T H$ showed CC, CT, and TT genotype, whereas A1819G polymorphism in the DBH had AA, GA, and GG genotype. These variable sequences were thought to cause amino acid substitution. The C97T and A1819G polymorphisms were found only in the Shiba Inu, suggesting that the polymorphic markers in the genes encoding catecholamine biosynthetic enzyme might be useful for examining the genetic background of behavioral characteristics in dog breeds (Takeuchi et al., 2005).

Analysis of polymorphic trinucleotide (CAG) repeat size (or number) in exon 1 of the $A R$ gene indicated that male dogs with a short allele had increased $A R$ function and showed higher aggressiveness scores than those with longer alleles (Konno et al., 2011). In vitro studies have demonstrated that the human $A R$ gene with a relatively short CAG repeat sequence possesses enhanced transcriptional activity by promoting the interaction between the receptor and co-activator (Chamberlain et al., 1994). The short CAG repeat sequences seem to be related to violent behavior and amygdala reactivity, which is involved in threat-related cues and presentations of angry faces (vs. happy) in human (Rajender et al., 2008; Manuck et al., 2010).

In addition, the microsatellite polymorphism could also act as a functional unit and interact with SNPs in the regulation of transcription. In human, an impact of microsatellite length on transcriptional activity was investigated in the background of haplotypes (rs35767:T > C, the CArepeat microsatellite, rs5742612:T > C, and rs2288377:T > A). As a form of T-(CA-repeat microsatellite)-C-A The
(CA) 17 repeat microsatellite with a C-T-T haplotype in the promoter region of the insulin-like growth factor 1 (IGF1) gene showed relatively low transcriptional activity, whereas the $(\mathrm{CA})_{21}$ repeat microsatellite with a T-C-A SNP, exhibited high transcriptional activity, suggesting that microsatellite length variation could control gene expression with a neighboring SNP event (Chen et al., 2013). While this study is limited to human genome, it demonstrates that analyses of microsatellite with SNP allow us to understand the genetic variability in dogs.

In case of microsatellite polymorphism and SNP, little has been known about its correlation with behavioral phenotype in dogs so far. However, the simple repeat microsatellites and SNPs have potential to be applied as molecular markers for each dog breed or individual identification. Gene-related microsatellites and SNPs could also be involved in functional gene regulation that results in different behavioral features.

\section{VNTR POLYMORPHISM OF FUNCTIONAL GENES IN DOGS}

Dogs have recently diverged and have maintained a relatively low genetic variability as a result of artificial selection and inbreeding. However, VNTR polymorphisms especially in neurotransmitter-related genes have been well studied in dog breeds (Hejjas et al., 2007, 2009; Ito et al., 2004; Kubinyi et al., 2012), and are summarized in Table 1. Aggressive behavior in dogs is a complex issue with various contributing aspects, including fear, activity level, and territoriality. The $D R D 4$ gene is believed to play a role in brain function and may influence affection and personality, and it is a candidate gene for behavior disorders. For example, allelic variants of the DRD4 gene are associated with novelty-seeking behavior in humans (Benjamin et al., 1996). The 24 bp insertion/ deletion in exon 1 of the $D R D 4$ gene described by Ito et al. (2004) was shown to be polymorphic in all dogs.

VNTR polymorphism in DRD4 exon 3 (a polymorphic region containing $39 \mathrm{bp}$ and $12 \mathrm{bp}$ units) was analyzed with respect to the behavior of German Shepherds, but it was difficult to elucidate behavior patterns (excitability, aggression, and impulsivity) or receptor function by allele frequencies of polymorphism (Niimi et al., 1999; Ito et al., 2004; Hejjas et al., 2007). A study addressing the molecular functional role and behavioral association of $D R D 4$ examined intron 2 of the canine $D R D 4$ gene. Behavioral analysis of 96 unrelated German Shepherds showed a significant association between the social impulsivity endophenotype of the Greeting Test and both the exonic and the intronic VNTRs of the DRD4 gene (Hejjas et al., 2009).

Recently, an association study was conducted between the $T H$ gene and the activity-impulsivity and inattention traits in a pet German Shepherd population. Dogs were 
Table 1. Published repeat polymorphism and SNP found in the behavior-associated functional genes in dogs

\begin{tabular}{|c|c|c|c|c|}
\hline Gene & Type & Location & Genotype & References \\
\hline AR (Androgen Receptor) & Microsatellite $^{1}$ & Exon 1 & (CAG)n & Konno et al., 2011 \\
\hline \multirow{2}{*}{ MAOA (Monoamine Oxydase A) } & Microsatellite & Exon 15 & $(\mathrm{TAAA}) \mathrm{n}$ & Klukowska et al., 2004 \\
\hline & Microsatellite & Exon 15 & $(\mathrm{GT}) \mathrm{n}$ & Klukowska et al., 2004 \\
\hline \multirow{2}{*}{ SLC1A2 (Solute Carrier Family 1 Member 2 ) } & \multirow{2}{*}{$\mathrm{SNP}^{2}$} & \multirow{2}{*}{ Coding region } & \multirow{2}{*}{$\mathrm{TT} / \mathrm{TC} / \mathrm{CC}$} & Takeuchi et al., 2009a \\
\hline & & & & Takeuchi et al., $2009 \mathrm{~b}$ \\
\hline TH (Tyrosine Hydroxylase) & SNP & Coding region & $\mathrm{CC} / \mathrm{CT} / \mathrm{TT}$ & Takeuchi et al., 2005 \\
\hline DBH (Dopamine b-Hydroxylase) & SNP & Coding region & AA/GA/GG & Takeuchi et al., 2005 \\
\hline \multirow{3}{*}{ DRD4 (Dopamine Receptor D4) } & VNTR & Exon 1 & $24 \mathrm{bp}$ & Ito et al., 2004 \\
\hline & VNTR & Exon 3 & 12 and $39 \mathrm{bp}$ & Niimi et al., 1999 \\
\hline & $\mathrm{VNTR}^{3}$ & Intron 2 & $17 \mathrm{bp}$ & Hejjas et al., 2009 \\
\hline \multirow{2}{*}{ TH (Tyrosine Hydroxylase) } & \multirow{2}{*}{$\mathrm{VNTR}^{4}$} & \multirow{2}{*}{ Intron 4} & \multirow{2}{*}{$36 \mathrm{bp}$} & Hejjas et al., 2007 \\
\hline & & & & Kubinyi et al., 2012 \\
\hline DBH (Dopamine $\beta$-Hydroxylase) & VNTR & Intron 4 & $17 \mathrm{bp}$ & Hejjas et al., 2007 \\
\hline DAT (Dopamine Transporter) & VNTR & Intron 9 & $38 \mathrm{bp}$ & Hejjas et al., 2007 \\
\hline DRD2 (Dopamine Receptor D2) & SINE & Intron 6 & tRNA-SINE(CA-polyA) & Jeoung et al., 2000 \\
\hline \multicolumn{5}{|c|}{$\begin{array}{l}{ }^{1} \text { Correlation between this polymorphic microsatellite and aggressiveness in Japanese Akita Inu has been reported (Takeuchi } \\
\text { et al., 2009b). } \\
2 \text { Correlation between this SNP and aggressiveness in Shiba Inu has been reported (Konno et al., 2011). } \\
3 \text { Correlation between this polymorphic VNTR and social impulsivity in German Shepherds has been reported (Hejjas et al., } \\
\text { 2009). } \\
4 \text { Correlation between this polymorphic VNTR and impulsivity in German Shepherds has been reported (Kubinyi et al., 2012). }\end{array}$} \\
\hline
\end{tabular}

assessed by the dog owners, who completed a questionnaire, and by experts, who scored the behavior of dogs in a battery of tests. The results suggested that a repeat polymorphism of $T H$ intron 4 was related to behavior in both the questionnaire and behavioral test (Kubinyi et al., 2012). Dogs carrying at least one short allele (a 36-bplong sequence) showed higher activity-impulsivity than those possessing two copies of the long allele (a duplicated form).

As shown in Table 1, the VNTR of a 17 bp segment in intron 4 of the $D B H$ gene was polymorphic in various dog breeds (Belgian Tervueren, Belgian Groenandael, Belgian Malinois, and German Shepherd) and the European Wolf. The copy number of the $17 \mathrm{bp}$ segment was either one or three; none of the examined samples were found to have a copy number of two (Hejjas et al., 2007). In the analysis, $38 \mathrm{bp}$ repetitive sequences were also identified in intron 9 of the DAT gene. German Shepherds possessed only the longer variant (allele 2), whereas Belgian Malinois showed an abundant form of the shorter variant (allele 1) compared to those of Belgian Tervuerens and Belgian Groenandaels (Hejjas et al., 2007). Furthermore, VNTR polymorphisms in $D R D 4, D B H$, and $D A T$ genes were shown to be associated with the activity-impulsivity phenotype among Belgian Tervuerens. Therefore, VNTR polymorphism of functional genes could be a molecular marker for attentive behavior in dog populations.

\section{SINE INSERTION AND GENOME DIVERSITY}

In the dog genome, protein-coding components represent about $1.5 \%$ of the total DNA, whereas $31 \%$ of the sequenced genome is composed of repetitive and mobile DNA elements (Kirkness et al., 2003). SINEs are capable of moving around in the genome, and can induce mutations, chromosomal rearrangements, and alterations to gene expression regulation. Therefore, these elements appear to be major factors in genomic diversity and evolution.

SINE elements are preferentially accumulated in Rbanding regions of the chromosome, whereas LINE and retroviral elements appear in G-banding regions. SINEs are derived from RNA polymerase III transcripts (7SL RNA and tRNA genes). The 7SL RNA-derived SINE elements are found only in humans, whereas tRNA-SINEs have been found in several species of the Canoidea, including the domestic dog (Canis familiaris) (Minnick et al., 1992). Occasionally, tRNA-SINEs are inserted into other repetitive DNA sequences such as LINEs or retroviral elements. In fact, $16 \%$ of tRNA-SINEs are inserted into LINEs in the dog genome. In this case, the SINEs are truncated at the insert end (Bentolila et al., 1999). Out of 483 clones possessing repetitive DNA sequences, 296 clones showed homology with tRNA-SINEs, which are mainly found in intronic regions or the $3^{\prime}$ UTR of canine genes (Bentolila et al., 1999). These findings sug- 
gest that SINE elements inserted in canine genome could be involved in gene regulation. In addition, IRS (interspersed repetitive DNA sequences) PCR analysis showed that canid IRS products are polymorphic (Das et al., 1998), suggesting that SINE insertion pattern could be developed as genetic markers for genotyping in dogs.

A SINE containing simple repeat sequences has been reported (Jeoung et al., 2000). The SINE element located in intron 6 of the canine D2 dopamine receptor (DRD2) gene has poly(A) sequences at the end and dinucleotide repeats (CT)8 (Jeoung et al., 2000) (Table 1). This study revealed a SINE in the DRD2 intron in German Shepherds (5 out of 5), Labrador Retrievers (3 out of 3 ), and a Beagle (1 out of 2 ). In 234 tested Sapsarees, this SINE was not detected in 151 individuals, while 80 individuals were found to carry it in heterozygous form, and 3 individuals were found to carry it in the homozygous form.

Polymorphic SINEs associated with functional genes are needed to be explored in dogs. Varying frequencies of SINEs in the dog population could provide allelespecific impacts on gene function among multiple dog breeds. In the genes related to behavioral traits, these polymorphisms could play essential roles in differential gene expression and phenotype depending on SINEs inserted in the genes.

\section{CONCLUSIONS AND FURTHER REMARKS}

In summary, we reviewed repeat elements including microsatellites, VNTRs, and SINEs in relation to functional genes (Table 1), suggesting that they have complex impacts on specific traits in dog breeds. Firstly, microsatellites related to behavior-related genes had a variable repeat size (or number) and affected behavioral characteristics in dog breeds (Takeuchi et al., 2009a, 2009b; Konno et al., 2011), and could also interact with SNPs in the regulation of transcription (Chen et al., 2013). Secondly, VNTRs of the dopaminergic neurotransmitter genes such as $D R D 4, T H, D B H$, and $D A T$ were reviewed in dogs (Hejjas et al., 2007, 2009), and a repeat polymorphism of the $T H$ gene was related to behavioral tests (Kubinyi et al., 2012). Lastly, canine SINEs, which are interspersed, present around every 5-8.3 kb (Das et al., 1998), are mainly found in intron or $3^{\prime}$ UTR of the genes in dogs. With respect to SINEs located in gene regulatory regions, it has been reported that SINEs in the DRD2 intron represented diversity in Sapsarees, German Shepherds, Labrador Retrievers, and a Beagle (Jeoung et al., 2000).

As described above, repeat elements located in various regions (exons, introns, 5' UTR, and 3' UTR) of the genes showed variation in multiple dog breeds, influencing gene regulation and consequently affecting phenotypes. As shown in Fig. 1, gene-associated repeat elements can be critical factors responsible for genetic variation and gene regulation through diverse mechanisms. These repeat elements could disrupt open reading frames through exonization or exon skipping, and could affect mRNA splicing events in coding regions. When located in the $5^{\prime}$ UTR or 3' UTR, repeat elements could also alter the level or pattern of gene expression by inserting promoter, enhancer, silencer, $\mathrm{CpG}$ island/methylation, terminator, or miRNA seed elements. This regulatory function could exert influence on variable phenotypes such as behavior in dog breeds.

These studies on repeat elements cast light on a new avenue for the investigation of gene regulation and phenotypic variation in complex traits. Thus, repeat elements should be examined in detail to reveal how the repeat polymorphism regulates genes and specific traits in dog breeds, such as behavioral variation and individual identification. More functional studies are needed to understand the role of polymorphic regulatory sequences located in and around functional genes with respect to gene expression.

This research was supported by awards from the AGENDA project (Project No. PJ009254) in the National Institute of Animal Science, Rural Development Administration (RDA).

\section{REFERENCES}

Bellizzi, D., Rose, G., Cavalcante, P., Covello, G., Dato, S., De Rango, F., Greco, V., Maggiolini, M., Feraco, E., Mari, V., et al. (2005) A novel VNTR enhancer within the SIRT3 gene, a human homologue of SIR2, is associated with survival at oldest ages. Genomics 85, 258-263.

Benjamin, J., Li, L., Patterson, C., Greenberg, B. D., Murphy, D. L., and Hamer, D. H. (1996) Population and familial association between the $\mathrm{D} 4$ dopamine receptor gene and measures of Novelty Seeking. Nat. Genet. 12, 81-84.

Bentolila, S., Bach, J. M., Kessler, J. L., Bordelais, I., Cruaud, C., Weissenbach, J., and Panthier, J. J. (1999) Analysis of major repetitive DNA sequences in the $\operatorname{dog}$ (Canis familiaris) genome. Mamm. Genome 10, 699-705.

Brunner, H. G., Nelen, M., Breakefield, X. O., Ropers, H. H., and van Oost, B. A. (1993) Abnormal behavior associated with a point mutation in the structural gene for monoamine oxidase A. Science 262, 578-580.

Chamberlain, N. L., Driver, E. D., and Miesfeld, R. L. (1994) The length and location of CAG trinucleotide repeats in the androgen receptor $\mathrm{N}$-terminal domain affect transactivation function. Nucleic Acids Res. 22, 3181-3186.

Chatterjee, S., and Pal, J. K. (2009) Role of 5'- and 3'-untranslated regions of mRNAs in human diseases. Biol. Cell 101, 251-262.

Chen, H. Y., Huang, W., Leung, V. H. K., Fung, S. L. M., Ma, S. L., Jiang, H., and Tang, N. L. S. (2013) Functional interaction between SNPs and microsatellite in the transcriptional regulation of insulin-like growth factor 1. Hum. Mutat. 34, 1289-1297.

Das, M., Chu, L. L., Ghahremani, M., Abrams-Ogg, T., Roy, M. S., Housman, D., and Pelletier, J. (1998) Characterization of an abundant short interspersed nuclear element (SINE) present in Canis familiaris. Mamm. Genome 9, 64-69.

Deckert, J., Catalano, M., Syagailo, Y. V., Bosi, M., Okladnova, O., Di Bella, D., Nöthen, M. M., Maffei, P., Franke, P., 
Fritze, J., et al. (1999) Excess of high activity monoamine oxidase A gene promoter alleles in female patients with panic disorder. Hum. Mol. Genet. 8, 621-624.

Ellegren, H. (2004) Microsatellites: simple sequences with complex evolution. Nat. Rev. Genet. 5, 435-445.

Gabellini, N. (2001) A polymorphic GT repeat from the human cardiac $\mathrm{Na}^{+} \mathrm{Ca}^{2+}$ exchanger intron 2 activates splicing. Eur. J. Biochem. 268, 1076-1083.

Hejjas, K., Vas, J., Kubinyi, E., Sasvari-Szekely, M., Miklosi, A., and Ronai, Z. (2007) Novel repeat polymorphisms of the dopaminergic neurotransmitter genes among dogs and wolves. Mamm. Genome 18, 871-879.

Hejjas, K., Kubinyi, E., Ronai, Z., Szekely, A., Vas, J., Miklosi, A., Sasvari-Szekely, M., and Kereszturi, E. (2009) Molecular and behavioral analysis of the intron 2 repeat polymorphism in the canine dopamine D4 receptor gene. Genes Brain Behav. 8, 330-336.

Irion, D. N., Schaffer, A. L., Famula, T. R., Eggleston, M. L., Hughes, S. S., and Pedersen, N. C. (2003) Analysis of genetic variation in $28 \mathrm{dog}$ breed populations with 100 microsatellite markers. J. Hered. 94, 81-87.

Ito, H., Nara, H., Inoue-Murayama, M., Shimada, M. K., Koshimura, A., Ueda, Y., Kitagawa, H., Takeuchi, Y., Mori, Y., Murayama, Y., et al. (2004) Allele frequency distribution of the canine dopamine receptor D4 gene exon III and I in 23 breeds. J. Vet. Med. Sci. 66, 815-820.

Jeoung, D., Myeong, H., Lee, H., Ha, J., Galibert, F., Hitte, C., and Park, C. (2000) A SINE element in the canine D2 dopamine receptor gene and its chromosomal location. Anim. Genet. 31, 334-335.

Kang, B. T., Kim, K. S., Min, M. S., Chae, Y. J., Kang, J. W., Yoon, J., Chol, J., Seong, J. K., Park, H. C., An, J., et al. (2009) Microsatellite loci analysis for the genetic variability and the parentage test of five dog breeds in South Korea. Genes Genet. Syst. 84, 245-251.

Kim, K. S., Tanabe, Y., Park, C. K., and Ha, J. H. (2001) Genetic variability in East Asian dogs using microsatellite loci analysis. J. Hered. 92, 398-403.

Kirkness, E. F., Bafna, V., Halpern, A. L., Levy, S., Remington, K., Rusch, D. B., Delcher, A. L., Pop, M., Wang, W., Fraser, C. M., et al. (2003) The dog genome: survey sequencing and comparative analysis. Science 301, 1898-1903.

Klukowska, J., Szczerbal, I., Wengi-Piasecka, A., Switonski, M., Schelling, C., Gmur, A., and Dolf, G. (2004) Identification of two polymorphic microsatellite in a canine BAC clone harbouring a putative canine MAOA gene. Anim. Genet. 35, $75-76$.

Konno, A., Inoue-Murayama, M., and Hasegawa, T. (2011) Androgen receptor gene polymorphisms are associated with aggression in Japanese Akita Inu. Biol. Lett. 7, 658-660.

Kubinyi, E., Vas, J., Hejjas, K., Ronai, Z., Brúder, I., Turcsán, B., Sasvari-Szekely, M., and Miklósi, A. (2012) Polymorphism in the tyrosine hydroxylase (TH) gene is associated with activity-impulsivity in German Shepherd Dogs. PLoS One 7, e30271.

Manuck, S. B., Marsland, A. L., Flory, J. D., Gorka, A., Ferrell, R. E., and Hariri, A. R. (2010) Salivary testosterone and a trinucleotide (CAG) length polymorphism in the androgen receptor gene predict amygdale reactivity in men. Psychoneuroendocrinology 35, 94-104.

Minnick, M. F., Stillwell, L. C., Heineman, J. M., and Stiegler, G. L. (1992) A highly repetitive DNA sequence possibly unique to canids. Gene 110, 235-238.

Niimi, Y., Inoue-Murayama, M., Murayama, Y., Ito, S., and Iwasaki, T. (1999) Allelic variation of the D4 dopamine receptor polymorphic region in two dog breeds, golden retriever and shiba. J. Vet. Med. Sci. 61, 1281-1286.

Parker, H. G., Kim, L. V., Sutter, N. B., Carlson, S., Lorentzen, T. D., Malek, T. B., Johnson, G. S., DeFrance, H. B., Ostrander, E. A., and Kruglyak, L. (2004) Genetic structure of the purebred domestic dog. Science 304, 1160-1164.

Payseur, B. A., Jing, P., and Haasl, R. J. (2011) A genomic portrait of human microsatellite variation. Mol. Biol. Evol. 28, 303-312.

Rajender, S., Pandu, G., Sharma, J. D., Gandhi, K. P. C., Singh, L., and Thangaraj, K. (2008) Reduced CAG repeats length in androgen receptor gene is associated with violent criminal behavior. Int. J. Legal Med. 122, 367-372.

Spady, T. C., and Ostrander, E. A. (2008) Canine behavioral genetics: pointing out the phenotypes and herding up the genes. Am. J. Hum. Genet. 82, 10-18.

Sun, J. X., Helgason, A., Masson, G., Ebenesersdóttir, S. S., Li, H., Mallick, S., Gnerre, S., Patterson, N., Kong, A., Reich, D., et al. (2012) A direct characterization of human mutation based on microsatellites. Nat. Genet. 44, 1161-1165.

Takeuchi, Y., Hashizume, C., Chon, E. M., Momozawa, Y. Masuda, K., Kikusui, T., and Mori, Y. (2005) Canine tyrosine hydroxylase (TH) gene and dopamine beta-hydroxylase $(\mathrm{DBH})$ gene: their sequences, genetic polymorphisms, and diversities among five different dog breeds. J. Vet. Med. Sci. 67, 861-867.

Takeuchi, Y., Hashizume, C., Arata, S., Inoue-Murayama, M., Maki, T., Hart, B. L., and Mori, Y. (2009a) An approach to canine behavioral genetics employing guide dogs for the blind. Anim. Genet. 40, 217-224.

Takeuchi, Y., Kaneko, F., Hashizume, C., Masuda, K., Ogata, N., Maki, T., Inoue-Murayama, M., Hart, B. L., and Mori, Y. (2009b) Association analysis between canine behavioral traits and genetic polymorphisms in the Shiba Inu bred. Anim. Genet. 40, 616-622.

Vonholdt, B. M., Pollinger, J. P., Lohmueller, K. E., Han, E., Parker, H. G., Quignon, P., Degenhardt, J. D., Boyko, A. R., Earl, D. A., Auton, A., et al. (2010) Genome-wide SNP and haplotype analyses reveal a rich history underlying dog domestication. Nature 464, 898-902. 